\title{
Electrostatic field in terms of geometric curvature in membrane MEMS devices
}

\author{
Paolo Di Barba $^{1}$, Luisa Fattorusso ${ }^{2}$, Mario Versaci ${ }^{3 *}$ \\ ${ }^{1}$ Dipartimento di Ingegneria Industriale e dell'Informazione, University of Pavia, \\ Pavia, Italy \\ ${ }^{2}$ Dipartimento di Ingegneria dell'Informazione Infrastrutture Energia Sostenibile, \\ "Mediterranea" University, Reggio Calabria, Italy \\ ${ }^{3}$ Dipartimento di Ingegneria Civile Energia Ambiente e Materiali, \\ "Mediterranea" University, Reggio Calabria, Italy \\ *Email address for correspondence: mario.versaci@unirc.it \\ Communicated by Nicola Bellomo \\ Received on 05 07, 2017. Accepted on 06 16, 2017.
}

\begin{abstract}
In this paper we present, in a framework of $1 D$-membrane Micro-Electro-MechanicalSystems (MEMS) theory, a formalization of the problem of existence and uniqueness of a solution related to the membrane deformation $u$ for electrostatic actuation in the steadystate case. In particular, we propose a new model in which the electric field magnitude $E$ is proportional to the curvature of the membrane and, for it, we obtain results of existence by Schauder-Tychonoff's fixed point application and subsequently we establish conditions of uniqueness. Finally, some numerical tests have been carried out to further support the analytical results.

Keywords: MEMS, NEMS, ELECTROSTATIC ACTUATION, BOUNDARY SEMI-LINEAR ELLIPTIC PROBLEMS, GREEN FUNCTION, FIXED-POINT THEOREM

AMS subject classification: 34B15, 34B27, 34B60, 34K30
\end{abstract}

\section{Introduction to the Problem}

In this last decade, the evolution of engineering application has been shifting more and more towards the embedded nature, to low-cost solutions, micro/nano dimensional, battery powered with multi-functional sensors and actuators playing a role of great importance because exploited as fundamental components to connect the physical nature of information with the abstract one represented in logic form in the machine. In such a context, it has gained the interest of the Scientific Community towards the modeling of Micro-Electro-Mechanical-Systems (MEMS). This interest is so felt in 


\section{P. DI BARBA, L. FATTORUSSO, M. VERSACI}

Science that in a recent survey, the MEMS technologies have been included among the twelve most promising technologies of the twenty-first century, destined to revolutionize the world of industry and consumer products and indicate as those technologies that will support more easily a new model of interface between man and electronic device. Born in 1964 with the production of the first batch device [1], the MEMS technology, starting as a purely engineering science, has increasingly turned into a physical-mathematical multi-discipline, thanks to advanced theoretical modeling request both in static and dynamic conditions, requiring soft skills highly specialized. However, the formulation of many theoretical models does not allow either to obtain explicit solutions, or the opportunity to prove their existence and uniqueness, nor any of their regularity property; for this reasons, under certain conditions, we derive implicit solutions to be studied only numerically. Scientific Community, in the MEMS domain, is busily working on two main fronts. The first one, purely theoretical, is heavily involved in modeling of coupled problems including the coupled thermal-elastic systems [2,3], the modeling of magnetically actuated systems $[4,5]$, the microfluidics devices [6-8] and the modeling of electric-elastic systems [9-11]. The second one, in practice, mainly deals with the MEMS technology transfer operational techniques in different areas pushing up to the modern Bio-MEMS for biomedical engineering, such as miniaturized bio-sensors and their application to diagnostics and tissue engineering $[12,13]$. In highly specialized fields such as modeling of static-magneto-thermo-elastic problems, for example, excellent results have been produced about the wave propagation in micro-domains with fixed boundary [14] and for the solution of inverse problems with moving boundary obtaining, in classical Hölder spaces, conditions that guarantee existence and uniqueness of the solution [15]. And yet, in transversely isotropic magneto-electro-elastic solid immersed in fluid it has introduced a particular decoupling technique exploiting particular potential functions [16]. Another line of research deals with the application of optinization theory in the automated design of MEMS: the design problem is formulated in terms of an inverse problem, like e.g. optimal shape synthesis, and then its solution is approximated by means of an algorithm of numerical minimization $[17,18]$. Some authors, in recent years, have gained expertise in the field of modeling of electrostatic actuators in MEMS in both steady cases and in the dynamical ones carrying out existence, uniqueness and regularity results by means of near operator theory even in presence of nonlinear singularities [19-22]. In these works, generalizing and deepening the research done in previous publications [23-27], it was considered a MEMS composed of two plates one of which is fixed and the other deformable, but clamped at boundary of a region $\Omega \in \mathbb{R}^{N}$; once a voltage 


\section{Electrostatic field in terms of geometric curvature in membrane MEMS devices}

drop is suitably applied, the ground plate deflects from the steady state $u=0$ towards a fixed plate (ground plate) positioned at height $u=1$. The deformation profile $u$ of the above mentioned $M E M S$, in the stationary case, was studied using the following model:

$$
\left\{\begin{array}{l}
\alpha \Delta^{2} u=\left(\beta \int_{\Omega}|\nabla u|^{2} d x+\gamma\right) \Delta u+\frac{\lambda_{1} f_{1}(x)}{(1-u)^{\sigma}\left(1+\chi \int_{\Omega} \frac{d x}{(1-u)^{\sigma-1}}\right)} \\
u=\Delta u-d u_{\nu}=0, \quad x \in \partial \Omega, \quad d \geq 0 \\
0<u<1, \quad x \in \Omega
\end{array}\right.
$$

where $f_{1}$ is a bounded function which carries dielectric properties of the material; $\lambda_{1}$ is the applied voltage between the ground plate and the deflecting plate; the positive parameters $\alpha, \beta, \gamma, \chi$ are related to the electric and mechanic properties of the material and with $\sigma \geq 2$ which takes into account more general potential than Coulomb's. This model is a generalization of the model studied in [23] in the limit case of zero ground plate thickness, hence for a thin plate with neglecting inertial effects as well as non-local effects, that is, with $\sigma=2$, as well as $\alpha=1, \beta=0, \gamma=0$, and $\chi=0$ (in dimensionless constants):

$$
\left\{\begin{array}{l}
\Delta^{2} u(x)=\frac{\lambda_{1} f_{1}(x)}{[1-u(x)]^{2}} \\
0<u(x)<1 \text { in } \Omega, \\
u=\Delta u-d u_{\nu}, \text { on } \partial \Omega, \quad d \geq 0
\end{array}\right.
$$

In this paper, starting from the above-mentioned works, and referring to the case where in place of the bottom plate we have a thin membrane attached to the edge and taking care, for an initial approach, of a $1 D$ model, we examine the following elliptical semi-linear model:

$$
\begin{cases}u^{\prime \prime}=-f_{2}(x) \frac{\lambda_{1}}{(1-u(x))^{2}} & \text { in } \Omega=[-L, L] \\ u=0 & \text { on } \partial \Omega .\end{cases}
$$

For the study of this model we propose a variant inspired by the consideration that the term $\lambda_{1}$, related to the voltage is, in fact, also related to the magnitude of the electric field $E$. Furthermore, since the electric field vector on the membrane is locally orthogonal to the tangent of the membrane, we have used in our model the fact that the magnitude of the electric field $E$ is proportional to the curvature $K$ of the membrane, finding a formulation of the problem in which the singularity due to $1-u(x)$ is not directly involved. The paper is organized as follows. Starting from a brief overview of the basic theoretical concepts for dimensionless electrostaticelastic systems (Section 2), and then presented the model related to the 


\section{P. DI BARBA, L. FATTORUSSO, M. VERSACI}

membrane systems, the authors propose the new approach based on the modeling problem (Section 3) in the Dirichlet's form also taking into account the safety distance $d^{*}$ of the membrane profile form the upper plate, then obtaining conditions ensuring existence and uniqueness (Section 4). To do this, the problem has been written in its integral formulation, through the use of a Green's function (Sections 5 and 6). Furthermore, some numerical verifications have been made in support of the proposed approach (Section 7).

\section{Theoretical Backgrounds}

We start from the general formulation of electrostatic-elastic MEMS systems with two parallel plates. Let us consider the ordinary space $\mathbb{R}^{3}$ and we fix a system of Cartesian axes $O^{\prime} x^{\prime} y^{\prime} z^{\prime}$ in it. In it, we consider an electrostatic-elastic system whose length is $2 L$ formed by a pair of parallel plates, of which one fixed and the other one elastic (but fixed at the edges), placed at a mutual distance $h$ with lying orthogonal to the axis z'. The system is subjected to an electric voltage $V$ : in particular, the elastic plate is subjected to the potential $V$ while the fixed one is subjected to the reference potential $V=0$ (see Fig. 1).

In such a context, the electrostatic potential $\phi$ satisfies the Lapalce's equation $\Delta \phi=0$ throughout the region bounded by the plates with boundary conditions $\phi=V$ on elastic plate and $\phi=0$ on fixed plate. With these assumptions, if we denote by $\Delta_{\perp}$ the Laplacian operator only with respect to $x^{\prime}$ and $y^{\prime}$, the deflection $w^{\prime}$ of the elastic plate satisfies the well-known following Equation [28]:

(4) $-\sigma \Delta_{\perp} w^{\prime}+D \Delta_{\perp}^{2} w^{\prime}=-\frac{\epsilon_{0}}{2}|\nabla \phi|^{2}$

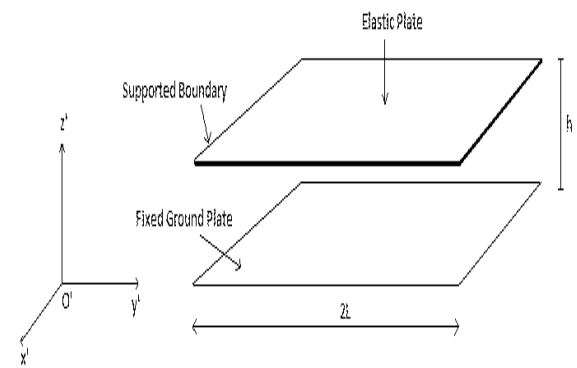

where $\sigma$ is the tension in the plate, $D$ is the flexural rigidity and $\epsilon_{o}$ is the perFigure 1. The electrostatic-elastic system: the elastic plate is at potential $V$, the fixed ground plate is at potential zero. mittivity of free space. We observe that in (4) there is a source term, proportional to the squared norm of the gradient of the potential, capturing the force on the plate due to the electric field coupling so the solution of the elastic problem to the solution of the electrostatic problem [29]. In order to re-frame the problem in dimensionless form, it is necessary to rescale the system. In particular, defining the 


\section{Electrostatic field in terms of geometric curvature in membrane MEMS devices}

following scaling factors:

$$
w=\frac{w^{\prime}}{h}, \Phi=\frac{\phi}{V}, x=\frac{x^{\prime}}{2 L}, y=\frac{y^{\prime}}{2 L}, \quad z=\frac{z^{\prime}}{h},
$$

and taking into account the Laplace's equation, (4) becomes a system of nonlinear coupled partial differential equations:

$$
\left\{\begin{array}{l}
\epsilon^{2} \Delta_{\perp} \Phi+\frac{\partial^{2} \Phi}{\partial z^{2}}=0 \\
-\Delta_{\perp} w+\delta \Delta_{\perp}^{2} w=-\lambda^{2}\left[\epsilon^{2}\left|\nabla_{\perp} \Phi\right|^{2}+\left(\frac{\partial \Phi}{\partial z}\right)^{2}\right] \\
\Phi=1 \text { on elastic plate } \\
\Phi=0 \text { on fixed plate }
\end{array}\right.
$$

in which $\delta$, relative importance of tension and rigidity, is $D /\left((2 L)^{2} \sigma\right), \epsilon$, the aspect ration of the system, is $h /(2 L)$ and, finally, $\lambda_{1}=\lambda^{2}$, the ratio of a reference electrostatic force to a reference elastic force, is:

$$
\lambda_{1}=\lambda^{2}=\frac{\epsilon_{0} V^{2}(2 L)^{2}}{2 h^{3} \sigma}=\beta V^{2}
$$

where, for each material the usual order of amplitude for each factor, we can write:

$$
\beta=\frac{\epsilon_{0}(2 L)^{2}}{2 h^{3} \sigma}
$$

represents the parameter which takes into account the electro-mechanical properties of the membrane material. It is worth observing that, in dimensionless conditions, $\beta$, taking into account (5), assumes the following new formulation:

$$
\beta_{1}=\frac{\epsilon_{0}}{2 \sigma}>10^{12}
$$

However, if we assume, as it is in the MEMS technology, that both thickness and width of the device are negligible with respect to the length $2 L,(6)$ assumes, as we see below, a greatly simplified form that, for many MEMS applications, is considered an excellent simplification. In particular, for $\epsilon \rightarrow$ 0 , the first equation of (6) assumes the form $\frac{\partial^{2} \Phi}{\partial z^{2}}=0$; if we solve the last one, taking into account the boundary conditions, we obtain the approximation of the potential $\Phi=\frac{z}{w}$, from which, the second equation of (6) assumes the non-linear form decoupled from the equation of the potential [28]:

$$
-\Delta_{\perp} w+\delta \Delta_{\perp}^{2} w=-\frac{\lambda^{2}}{w^{2}}
$$




\section{P. DI BARBA, L. FATTORUSSO, M. VERSACI}

hardly solvable except for sporadic cases characterized by simple geometries. The MEMS model we propose to study is one in which the deformable plate shown in Figure 1 is replaced by a deformable membrane anchored along the edge of the lower face of a fixed plate. First, we note that the fixed plate has the exclusive task of supporting the deformable membrane for which, studying the deflection of the membrane due to the application of the potential difference to the plates, it is not considered and, in addition (10) is still valid even in the case of the replacement of the elastic plate by the membrane (obviously with different values of the parameters which take into account the electro-mechanical properties of the membrane). However, the new techniques of production of membranes in industrial domain allow for the exploitation of materials with flexural rigidity $D$ negligible for which (10) can be simplified by imposing $\delta=0$; therefore, in steady-state deflection conditions, the problem associated to (10) assumes the following semi-linear elliptic form:

$$
\left\{\begin{array}{l}
\frac{d^{2} w}{d x^{2}}=\frac{\lambda^{2}}{w^{2}} \text { in } \Omega \\
w(-1 / 2)=w(1 / 2)=1 .
\end{array}\right.
$$

From (11), by placing $w=1+u$, we obtain the following homogeneous problem:

$$
\left\{\begin{array}{l}
\frac{d^{2} u}{d x^{2}}=\frac{\lambda^{2}}{(1+u)^{2}} \text { in } \Omega \\
u(-1 / 2)=u(1 / 2)=0
\end{array}\right.
$$

that, reversing the orientation of $z$ so that the membrane in the rest position lies on $z=0$, can be written as:

$$
\widetilde{P}:\left\{\begin{array}{l}
\frac{d^{2} u}{d x^{2}}=-\frac{\lambda^{2}}{(1-u)^{2}} \text { in } \Omega \\
u(-1 / 2)=u(1 / 2)=0
\end{array}\right.
$$

that is the problem we are going to study. In addition, for simplicity, we indicate by $L_{1}=1 / 2$ the rescaled semi-length of the device (13) $L_{1}=1 / 2$ [28].

\section{The Proposed Approach}

Starting from the equation associated with the problem $\widetilde{P}$, now we present an innovative approach considering the magnitude of the electric field $E$ proportional to the curvature of the membrane, taking into account that the electric field vector on the membrane is locally orthogonal to the tangent to the membrane. Therefore, we consider the problem $\widetilde{P}$ : in it $\lambda^{2}$, 


\section{Electrostatic field in terms of geometric curvature in membrane MEMS devices}

taking into account thT (7) is proportional to the square of the electric voltage, for which the term $\frac{\lambda^{2}}{(1-u)^{2}}$ is proportional to the square of the magnitude of the electric field $E$. Therefore, $\widetilde{P}$ is rewritable in the following form:

$$
\widetilde{\widetilde{P}}:\left\{\begin{array}{l}
-u^{\prime \prime}=\beta_{1} E^{2} \text { in } \Omega=\left[-L_{1}, L_{1}\right] \\
u\left(-L_{1}\right)=u\left(L_{1}\right)=0
\end{array}\right.
$$

where $\beta_{1}$ is the coefficient of proportionality defined in (9) taking into account the electro-mechanical properties of the material constituting the membrane. Since $E^{2}$ in (14) represents the square of the electric field magnitude and keeping in mind that, from the electrostatic point of view, the lines of force of the electric field vector are orthogonal, point by point, to the tangent of the membrane surface, we can express $E$ as the product between a coefficient of proportionality $\mu$ and the curvature $K$ of the membrane deflection. This approach has also been tested on a well-known hemispherical benchmark in literature [30] from which it was highlighted the dependence of $\mu$ on both $x$ and $u(x)$; therefore:

$$
E(x)=\mu(x, u(x), \lambda) K(x, u(x)) .
$$

In addition, since from the electrostatic point of view it is unacceptable the condition for which the deflection of the membrane touches the upper plate of the device (situation mathematically representable by a singularity), with good reason, we can also think with reference to the previously studied models, that:

$$
\mu(x, u(x), \lambda)=\frac{\lambda}{1-u(x)}
$$

with $\mu(x, u(x)) \in C^{0}(A), \quad A=[-L, L] \times[0,1)$. To prevent the condition of electric discharge in the dielectric material filling the region between the two plates, it is necessary that the membrane is sufficiently distant from the upper plate; we will put:

$$
\mu(x, u(x), \lambda)=\frac{\lambda}{1-u(x)-d^{*}}
$$

with $d^{*}$ critical distance equal to $\frac{\lambda}{\epsilon_{t}}$, with $\epsilon_{t}$ dielectric strength of the material constituting the membrane, even when the deflection $u$ assumes its maximum deformation. Obviously, in the case of materials with ideal behaviour, $\epsilon_{t}$ tends to infinity so that you restore the model (13). Finally, $\widetilde{\widetilde{P}}$ 


\section{P. DI BARBA, L. FATTORUSSO, M. VERSACI}

assumes the following form:

(18)

$\widetilde{\widetilde{P}}:\left\{\begin{array}{l}-u^{\prime \prime}=\beta_{1} \mu^{2}(x, u(x), \lambda) K^{2}(x, u(x))=\beta_{1} \lambda^{2} \frac{K^{2}(x, u(x))}{\left(1-u(x)-d^{*}\right)^{2}} \text { in } \Omega=\left(-L_{1}, L_{1}\right) \\ u\left(-L_{1}\right)=u\left(L_{1}\right)=0 \\ 0<u(x)<1-d^{*} .\end{array}\right.$

Since, in $1 D$ regimes, the curvature $K$ is expressed by

$$
K(x, u(x))=\frac{\left|u^{\prime \prime}(x)\right|}{\sqrt{\left(1+\left|u^{\prime}(x)\right|^{2}\right)^{3}}},
$$

from the new version of $\widetilde{\widetilde{P}}$ we can write the associated equation:

$$
u^{\prime \prime}(x)+\beta \frac{\mu^{2}(x, u(x), \lambda)\left|u^{\prime \prime}(x)\right|^{2}}{\left(1+\left(u^{\prime}(x)\right)^{2}\right)^{3}}=0 .
$$

From which, taking into account that $u(x)>0$, we can have the two following cases:

- $u^{\prime \prime}(x)=0$ that is $u^{\prime}(x)=k$ with $k$ arbitrary constant; in this case the deflection of the membrane is linear, that is $u(x)=k x+b$. This condition is unwrapped because from $u^{\prime \prime}(x)=0$ would follow $E=0$, producing a linear deflection of the membrane (physically impossible occurrence); or:

$$
\left(1+\beta_{1} \frac{\mu^{2}(x, u(x), \lambda)\left(u^{\prime \prime}(x)\right)}{\left(1+\left(u^{\prime}(x)\right)^{2}\right)^{3}}\right)=0
$$

from which $\widetilde{\widetilde{P}}$, taking into account (17), becomes:

$$
\widetilde{\widetilde{P}}:\left\{\begin{array}{l}
u^{\prime \prime}(x)=-\frac{\left(1+\left(u^{\prime}(x)\right)^{2}\right)^{3}}{\beta_{1} \mu^{2}(x, u(x), \lambda)} \text { in } \Omega=\left[-L_{1}, L_{1}\right] \\
u\left(-L_{1}\right)=u\left(L_{1}\right)=0 \\
0<u(x)<1-d^{*}
\end{array}\right.
$$

\section{General Formulation of the Problem}

We can study $\widetilde{\widetilde{P}}$ as a special case of the following general problem. Let us consider $\Omega=\left(-L_{1}, L_{1}\right), u: \Omega \rightarrow \mathbb{R}$. We suppose, as it is well-known, that $u \in C^{2}(\bar{\Omega})$ is the solution of the following general Dirichlet's problem:

$$
\text { Problem } I:\left\{\begin{array}{l}
u^{\prime \prime}(x)+f\left(x, u(x), u^{\prime}(x)\right)=0 \text { in } \Omega \\
u\left(-L_{1}\right)=u\left(L_{1}\right)=0 \\
0<u<\alpha
\end{array}\right.
$$


with $f \in C^{0}(\bar{\Omega} \times \mathbb{R} \times \mathbb{R})$ and $\alpha=1-d^{*}$; assuming

$$
f\left(x, u(x), u^{\prime}(x)\right)=\frac{1}{\beta_{1}} \frac{\left(1+\left(u^{\prime}(x)\right)^{2}\right)^{3}}{\mu^{2}(x, u(x), \lambda)},
$$

Problem I can be written as follows:

Problem $I:\left\{\begin{array}{l}u^{\prime \prime}=-\frac{\left(1+\left(u^{\prime}(x)\right)^{2}\right)^{3}}{\beta_{1} \mu^{2}(x, u(x), \lambda)}=-\frac{1}{\beta_{1} \lambda^{2}}\left(1+\left(u^{\prime}(x)\right)^{2}\right)^{3}(\alpha-u(x))^{2} \text { in } \Omega \\ u\left(-L_{1}\right)=u\left(L_{1}\right)=0 \\ 0<u<\alpha\end{array}\right.$

with $\left.u \in C^{2}\left(\left[-L_{1}, L_{1}\right]\right){ }^{\mathrm{a}}\right), \mu=\mu(x, u(x), \lambda) \in C^{0}\left(\left[-L_{1}, L_{1}\right] \times \mathbb{R}\right)$ where $\mu=\frac{\lambda}{\alpha-u(x)}$. Apparently Problem I, as formulated in (25), does not present the singularity characterizing (13), or in our case when $u=1-d^{*}$ in (18). In this eventuality, from the equation associated to (25), we would get $u^{\prime \prime}(x)=0$ that, as pointed out previously, would provide $E=0$ producing a linear deflection of the membrane (physically unacceptable condition).

\section{A Result of Existence for Problem $I$}

We start premising two definitions.

Definition 5.1. Let $P$ be the functional space so defined:

$$
P=\left\{C_{0}^{2}\left[-L_{1}, L_{1}\right]: \quad 0<u(x)<\alpha,\left|u^{\prime}(x)\right|<H\right\}
$$

Definition 5.2. Let $P_{1}$ be the functional space defined as follows:

$$
P_{1}=\left\{C_{0}^{1}\left[-L_{1}, L_{1}\right]: 0<u(x)<\alpha,\left|u^{\prime}(x)\right|<H\right\}^{\mathrm{b}}
$$

It is well-know [31] that (23), by differentiation, can be transformed in the following integral equation:

$$
u(x)=\int_{-L_{1}}^{L_{1}} G(x, s) f\left(s, u(s), u^{\prime}(s)\right) d s
$$

with $0<u<\alpha$ and $G(x, s)$ a suitable Green's function.

It is also known that:

$$
u^{\prime}(x)=\int_{-L_{1}}^{L_{1}} G_{x}(x, s) f\left(s, u(s), u^{\prime}(s)\right) d s
$$

\footnotetext{
${ }^{a}$ as well-known, this assumption is physically plausible because membrane tears are not allowed and slopes vary continuously.
} 


\section{P. DI BARBA, L. FATTORUSSO, M. VERSACI}

in particular, in our case, (25) is transformed in the following integral equation:

$$
u(x)=\int_{-L_{1}}^{L_{1}} G(x, s) \frac{\left(1+\left(u^{\prime}(s)\right)^{2}\right)^{3}}{\beta_{1} \mu^{2}(s, u(s), \lambda)} d s .
$$

We can then prove the existence of the solution of the equation $T(u)=w$, with $u \in P_{1}$, exploiting the Schauder-Tychonoff fixed point theorem applied to the operator $w=T(u)$ from $P$ to $P$. In fact, from (30), it is possible to define the positive operator $T$ as follows:

$$
T(u(x))=\int_{-L_{1}}^{L_{1}} G(x, s) \frac{\left(1+\left(u^{\prime}(s)\right)^{2}\right)^{3}}{\beta_{1} \mu^{2}(s, u(s), \lambda)} d s
$$

and from which:

$$
T^{\prime}(u(x))=\int_{-L_{1}}^{L_{1}} G_{x}(x, s) \frac{\left(1+\left(u^{\prime}(s)\right)^{2}\right)^{3}}{\beta_{1} \mu^{2}(s, u(s), \lambda)} d s .
$$

The Green's function here exploited for our purposes is [31]:

$$
G(x, s)= \begin{cases}\frac{\left(s+L_{1}\right)\left(L_{1}-x\right)}{2 L_{1}} & -L_{1} \leq s \leq x \\ \frac{\left(L_{1}-s\right) \cdot\left(x+L_{1}\right)}{2 L_{1}} & x \leq s \leq L_{1}\end{cases}
$$

from which we obtain:

$$
G_{x}(x, s)= \begin{cases}\frac{-\left(s+L_{1}\right)}{2 L_{1}} & -L_{1} \leq s \leq x \\ \frac{\left(L_{1}-s\right)}{2 L_{1}} & x \leq s \leq L_{1}\end{cases}
$$

The following properties for $G$ are particularly useful.

1. $G(x, s)$ is a continuous and non-negative function;

2. $G(x, s)$ has its maximum equal to $L_{1} / 2$ on the straight line $x=s$ at $s=0$. So, we can write:

$$
0 \leq G(x, s) \leq L_{1} / 2 \quad \forall x, s \in\left[-L_{1}, L_{1}\right] ;
$$

3. we can easily calculate its integral over $[-L, L]$ as follows:

$$
\begin{aligned}
\int_{-L_{1}}^{L_{1}} G(x, s) d s=\frac{L_{1}-x}{2 L_{1}} \int_{-L_{1}}^{x}\left(s+L_{1}\right) d s & +\frac{x+L_{1}}{2 L_{1}} \int_{x}^{L_{1}}\left(L_{1}-s\right) d s= \\
& =\frac{\left(L_{1}-x\right)\left(x+L_{1}\right)}{2} \leq \frac{L_{1}^{2}}{2}
\end{aligned}
$$




\section{Electrostatic field in terms of geometric curvature in membrane MEMS devices}

4. we can calculate the integral of $\left|G_{x}(x, s)\right|$ over $\left[-L_{1}, L_{1}\right]$ :

$$
\left|\int_{-L_{1}}^{L_{1}} G_{x}(x, s) d s\right| \leq \int_{-L_{1}}^{L_{1}}\left|G_{x}(x, s)\right| d s \leq \frac{2 L_{1}}{2}=L_{1}
$$

5. finally, $\forall x, s \in\left(\left[-L_{1}, L_{1}\right] \times\left[-L_{1}, L_{1}\right]\right)$ :

$$
G_{x}(x, s) \leq \frac{1}{2}
$$

In order to apply the Schauder-Tychonoff fixed-point theorem, we prove the following result.

Theorem 5.1. Consider the operator $T(u)$ defined by (31). It is an operator from $P$ to $P$.

Proof. Taking into account the definition of the $C^{2}$-norm, to prove that $T(u) \in C_{0}^{2}\left(\left[-L_{1}, L_{1}\right]\right)$, we must prove that:

$$
\begin{aligned}
\left.|| T(u(x))\right|_{C^{2}\left[-L_{1}, L_{1}\right]}= & \sup _{x \in\left[-L_{1}, L_{1}\right]}|T(u(x))|+\sup _{x \in\left[-L_{1}, L_{1}\right]}\left|T^{\prime}(u(x))\right| \\
& +\sup _{x \in\left[-L_{1}, L_{1}\right]}\left|T^{\prime \prime}(u(x))\right|<+\infty .
\end{aligned}
$$

Preliminarily, we observe that $T\left(u\left(-L_{1}\right)\right)=T\left(u\left(L_{1}\right)\right)=0$ for construction of $G(x, s)$ and one can easily verify that $T(u) \geq 0$. We observe that, from the formulation of Problem $I$, and particularly from (17), we can infer that $\mu(x, u(x))>1$ in $\left[-L_{1}, L_{1}\right]$. Such condition is physically confirmed by the fact that $E$, to deform the membrane, must locally win the inertia of deformation for which $\mu(x, u(x))$, which represent the coefficient of proportionality between $E$ and curvature $K$, necessarily assumes values greater than the unity. Then, depending on the material, to overcome the intertia of the membrane, a minimum voltage $\bar{\lambda}>0$ must be applied so that $\bar{\lambda}<\lambda<\sup \{\lambda\}^{\mathrm{c}}$ ensuring that $1 / \lambda^{2}<+\infty$. Then, taking into account

\footnotetext{
${ }^{c}$ Obviously, $\sup \{\lambda\}$ is a bounded quantity depending on the kind of the device.
} 


\section{P. DI BARBA, L. FATTORUSSO, M. VERSACI}

also the (35), we can write:

$$
\begin{array}{r}
0 \leq|T(u(x))| \leq \sup _{x \in\left[-L_{1}, L_{1}\right]}|T(u(x))|= \\
=\sup _{x \in\left[-L_{1}, L_{1}\right]}\left|\int_{-L_{1}}^{L_{1}} G(x, s) \frac{\left(1+\left(u^{\prime}(s)\right)^{2}\right)^{3}}{\beta_{1} \mu^{2}} d s\right|=
\end{array}
$$

$\leq \frac{1}{\beta_{1} \lambda^{2}} \sup _{x \in\left[-L_{1}, L_{1}\right]}\left|\int_{-L_{1}}^{x} \frac{\left(s+L_{1}\right)\left(L_{1}-x\right)}{2 L_{1}}\left(1+\left(u^{\prime}(s)\right)^{2}\right)^{3}(\alpha-u(s))^{2} d s\right|+$ $+\frac{1}{\beta_{1} \lambda^{2}} \sup _{x \in\left[-L_{1}, L_{1}\right]}\left|\int_{x}^{L} \frac{\left(L_{1}-s\right)\left(x+L_{1}\right)}{2 L_{1}}\left(1+\left(u^{\prime}(s)\right)^{2}\right)^{3}(\alpha-u(s))^{2} d s\right|=$ $=\alpha \frac{1}{\beta_{1} \lambda^{2}}\left\{\sup _{x \in\left[-L_{1}, L_{1}\right]} \mid \int_{-L_{1}}^{x} \frac{\left(s+L_{1}\right)\left(L_{1}-x\right)}{2 L_{1}}\left(1+\left(u^{\prime}(s)\right)^{2}\right)^{3} d s+\right.$ $\left.+\int_{x}^{L_{1}} \frac{\left(L_{1}-s\right)\left(x+L_{1}\right)}{2 L_{1}}\left(1+\left(u^{\prime}(s)\right)^{2}\right)^{3} d s \mid\right\} \leq$ $\leq 4 \alpha \frac{1}{\beta_{1} \lambda^{2}}\left(1+H^{6}\right) \sup _{x \in\left[-L_{1}, L_{1}\right]}\left\{\int_{-L_{1}}^{x} \frac{\left(s+L_{1}\right)\left(L_{1}-x\right)}{2 L_{1}} d s+\right.$ $\left.\int_{x}^{L_{1}} \frac{\left(L_{1}-s\right)\left(x+L_{1}\right)}{2 L_{1}} d s\right\} \leq 4 \alpha \frac{1}{\beta_{1} \lambda^{2}}\left(1+H^{6}\right) L_{1}^{2}<+\infty$.

In addition:

$(41)$

$$
\begin{array}{r}
\sup _{x \in\left[-L_{1}, L_{1}\right]}\left|T^{\prime}(u(x))\right|=\sup _{x \in\left[-L_{1}, L_{1}\right]}\left|\int_{-L_{1}}^{L_{1}} G_{x}(x, s) \frac{\left(1+\left(u^{\prime}(s)\right)^{2}\right)^{3}}{\beta_{1} \mu^{2}} d s\right|= \\
=\frac{1}{\beta_{1} \lambda^{2}} \sup _{x \in\left[-L_{1}, L_{1}\right]} \mid \int_{-L_{1}}^{x}-\frac{s+L_{1}}{2 L_{1}}\left(1+\left(u^{\prime}(s)\right)^{2}\right)^{3}(\alpha-u(s))^{2} d s+ \\
+\int_{x}^{L_{1}}-\frac{L_{1}-s}{2 L_{1}}\left(1+\left(u^{\prime}(s)\right)^{2}\right)^{3}(\alpha-u(s))^{2} d s \mid< \\
\leq 4 \alpha \frac{1}{\beta_{1} \lambda^{2}}\left(1+H^{6}\right) \sup _{x \in\left[-L_{1}, L_{1}\right]}\left|\int_{-L_{1}}^{x}-\frac{s+L_{1}}{2 L_{1}} d s+\int_{x}^{L_{1}} \frac{L_{1}-s}{2 L_{1}} d s\right| \leq \\
\leq 4 \alpha \frac{1}{\beta_{1} \lambda^{2}}\left(1+H^{6}\right) L_{1}<+\infty .
\end{array}
$$

Therefore, taking into account the (32), the (34), the (38), that $\left|u^{\prime}\right| \leq H$ and $\left|1 / \mu^{2}\right|<1$, we easily obtain: 


$$
\begin{array}{r}
\sup _{x \in\left(\left[-L_{1}, L_{1}\right]\right)}\left|T^{\prime \prime}(u(x))\right|= \\
=\sup _{x \in\left(\left[-L_{1}, L_{1}\right]\right)}\left|\frac{d}{d x} \int_{-L_{1}}^{L_{1}} G_{x}(x, s)\left(\frac{\left(1+\left(u^{\prime}(s)\right)^{2}\right)^{3}}{\beta_{1} \mu^{2}}\right) d s\right|= \\
\leq \frac{1}{2 \beta_{1} \lambda^{2}} \sup _{x \in\left(\left[-L_{1}, L_{1}\right]\right)}\left|\left(\frac{\left(1+\left(u^{\prime}(s)\right)^{2}\right)^{3}}{\beta_{1} \mu^{2}}\right)\right|+ \\
+\frac{1}{2 \beta_{1} \lambda^{2}} \sup _{x \in\left(\left[-L_{1}, L_{1}\right]\right)}\left|\left(\frac{\left(1+\left(u^{\prime}(s)\right)^{2}\right)^{3}}{\beta_{1} \mu^{2}}\right)\right| \leq \\
\leq\left(\frac{1}{2 \beta_{1}}+\frac{1}{2 \beta_{1}}\right) \frac{\left(1+H^{2}\right)^{3}}{\lambda^{2}}=\frac{1}{\beta_{1}} \frac{\left(1+H^{2}\right)^{3}}{\lambda^{2}}<+\infty .
\end{array}
$$

Substituting (40), (41) and (42) into (39), we can infer that:

$$
\begin{gathered}
\|T(u(x))\|_{C^{2}\left(\left[-L_{1}, L_{1}\right]\right)} \leq 4 \alpha \frac{1}{\beta_{1} \lambda^{2}}\left(1+H^{6}\right) L_{1}^{2}+ \\
+4 \alpha \frac{1}{\beta_{1} \lambda^{2}}\left(1+H^{6}\right) L_{1}+\frac{1}{\beta_{1} \lambda^{2}}\left(1+H^{2}\right)^{3}<+\infty .
\end{gathered}
$$

Now we show that $T(u) \in P$. From (42), to obtain that $T(u) \in P$, we must have $4 \alpha \frac{1}{\beta_{1} \lambda^{2}}\left(1+H^{6}\right) L_{1}^{2}<\alpha$, from which:

$$
1+H^{6}<\frac{\beta_{1} \overline{\lambda^{2}}}{4 L_{1}^{2}} \Rightarrow H<\sqrt[6]{\frac{\beta_{1} \overline{\lambda^{2}}}{4 L_{1}^{2}}-1 .}
$$

Therefore, having being true both (41) and (44), should be verified the following system:

$$
\left\{\begin{array}{l}
1+H^{6}<\frac{H \beta_{1} \overline{\lambda^{2}}}{4 \alpha L_{1}} \\
1+H^{6}<\frac{\beta_{1} \bar{\lambda}^{2}}{4 L_{1}^{2}}
\end{array}\right.
$$

To compare the second members of (45) d , we suppose, by contradiction, that $\frac{\beta_{1} \overline{\lambda^{2}}}{4 L_{1}^{2}}<\frac{H \beta_{1} \overline{\lambda^{2}}}{4 \alpha L_{1}}$ and from this we would get $H>\frac{\alpha}{L_{1}}=2 \alpha$. But, taking into account the scaling factors (5) and the fact that $H=\frac{z}{x}$ and $H^{\prime}=\frac{z^{\prime}}{x^{\prime}}$, we can write:

$$
H=\frac{z}{x}=\frac{z^{\prime}}{h} \frac{2 L}{x^{\prime}}=H^{\prime} \frac{2 L}{h}>2 \alpha .
$$

\footnotetext{
${ }^{d}$ taking into account that the sixth degree inequalities do not allow resolution by radicals
} 


\section{P. DI BARBA, L. FATTORUSSO, M. VERSACI}

But $\alpha=1-d^{*}$ that, in no-scaling conditions, becomes $\alpha=\frac{\alpha^{\prime}}{h}$ and taking into account (46) we obtain $H^{\prime}>\frac{\alpha^{\prime}}{L}$. Because $L$ is the semi-length of the wafer without scaling so, therefore, if $L \rightarrow 0, \frac{\alpha^{\prime}}{L} \rightarrow+\infty$ and being $\alpha^{\prime}$ a positive limited coefficient, then $H^{\prime}=\sup \left|u^{\prime}\right|$ would be greater than a quantity tending to $+\infty$ inconsistently with the membership of $u$ to $C^{2}\left(\left[-L_{1}, L_{1}\right]\right)$. So $\frac{\beta_{1} \overline{\lambda^{2}}}{4 L_{1}^{2}}>\frac{H \beta_{1} \overline{\lambda^{2}}}{4 \alpha L_{1}}$ holds from which (45) is equivalent to the only inequality:

$$
1+H^{6}<\frac{H \beta_{1} \overline{\lambda^{2}}}{4 \alpha L_{1}}
$$

which represents the condition guaranteeing that $T(u): P \rightarrow P$.

Theorem 5.2. Problem I admits at least one solution in $P$.

Proof. Considering the result obtained by the Theorem 5.1 and since the compact immersion $C_{0}^{2}\left[-L_{1}, L_{1}\right] \hookrightarrow C_{0}^{1}\left[-L_{1}, L_{1}\right]$ holds and, again, the compact immersion $P_{1} \hookrightarrow P$ holds, then, applying the Schauder-Tychonoff fixed-point theorem, the problem $u=T(w)$ admits at least a fixed point $u=T(u)$ in $P_{1}$ that is there exists at least a solution for Problem $I$.

Observing (47) we can say that not only we have shown a condition of existence but we have highlighted that this condition depends on the properties of the material constituting the membrane (presence of $\beta$ ).

\section{On the uniqueness of the Solution for Problem I}

Let us consider Problem $I$.

Theorem 6.1. $\forall H>0$ the solution of the Problem I is unique. In addition, the following properties hold:

1) $\forall x \in[-L, L],\left|u^{\prime}(x)\right| \leq\left|u^{\prime}(L)\right|=\left|u^{\prime}(-L)\right|$;

2) $u$ is symmetric with respect to the origin;

3) $u \in C^{\infty}([-L, L])$;

4) $u$ is analytical.

We begin the proof showing 1). From the equation of Problem I, we infer that, being $u$ " $(x) \leq 0$ in $[-L, L]$ the solution $u$ is concave over the same interval and its first derivative is decreasing. In addition, the above mentioned equation can be written as follows:

$$
\frac{u "(x)}{\left[1+\left(u^{\prime}(x)\right)^{2}\right]^{3}}=-\frac{1}{\beta_{1} \overline{\lambda^{2}}}\left[1-d^{*}-u(x)\right]^{2} .
$$




\section{Electrostatic field in terms of geometric curvature in membrane MEMS devices}

Multiplying by $u^{\prime}$ both member of (48), we obtain:

$$
\begin{array}{r}
\frac{u^{\prime \prime}(x) u^{\prime}(x)}{\left[1+\left(u^{\prime}(x)\right)^{2}\right]^{3}}=-\frac{1}{\beta_{1} \overline{\lambda^{2}}}\left[1-d^{*}-u(x)\right]^{2} u^{\prime}(x)= \\
=-\frac{1}{\beta_{1} \overline{\lambda^{2}}}(1-d *)^{2} u^{\prime}(x)+\frac{1}{\beta_{1} \overline{\lambda^{2}}}\left(1-d^{*}\right) \frac{d}{d x}[u(x)]^{2}-\frac{1}{3 \beta_{1} \overline{\lambda^{2}}} \frac{d}{d x}[u(x)]^{3} .
\end{array}
$$

Because

$$
\frac{u^{\prime \prime}(x) u^{\prime}(x)}{\left[1+\left(u^{\prime}(x)\right)^{2}\right]^{2}}=-\frac{1}{4} \frac{d}{d x} \frac{1}{\left(1+\left[u^{\prime}(x)\right]^{2}\right)^{2}}
$$

by integration from $-L$ to $L,(49)$, we obtain

$$
-\frac{1}{4} \frac{1}{\left(1+\left[u^{\prime}(L)\right]^{2}\right)^{2}}+\frac{1}{4} \frac{1}{\left(1+\left[u^{\prime}(-L)\right]^{2}\right)^{2}}=0
$$

from which $\left|u^{\prime}(-L)\right|=\left|u^{\prime}(L)\right|$. Moreover, by integration of (49) from $-L$ to $t$, taking into account that $u(-L)=0$ and that $\forall t \in[-L, L]$ we can write:

$$
\begin{array}{r}
-\frac{1}{4} \frac{1}{\left(1+\left[u^{\prime}(t)\right]^{2}\right)^{2}}+\frac{1}{4} \frac{1}{\left(1+\left[u^{\prime}(-L)\right]^{2}\right)^{2}}= \\
=-\frac{1}{\beta_{1} \overline{\lambda^{2}}}(1-d *)^{2} u(t)+\frac{1}{\beta_{1} \overline{\lambda^{2}}}\left(1-d^{*}\right)[u(t)]^{2}-\frac{1}{3 \beta_{1} \overline{\lambda^{2}}}[u(t)]^{3},
\end{array}
$$

and then $\forall t \in[-L, L]$

$$
\begin{array}{r}
-\frac{1}{\beta_{1} \overline{\lambda^{2}}}(1-d *)^{2} u(t)+\frac{1}{\beta_{1} \overline{\lambda^{2}}}\left(1-d^{*}\right)[u(t)]^{2}-\frac{1}{3 \beta_{1} \overline{\lambda^{2}}}[u(t)]^{3}= \\
=\frac{1}{\beta_{1} \overline{\lambda^{2}}} u(t)\left\{(1-d *)\left[u(t)-\left(1-d^{*}\right)\right]-\frac{1}{3}[u(t)]^{2}\right\}<0
\end{array}
$$

we deduce that:

$$
-\frac{1}{4} \frac{1}{\left(1+\left[u^{\prime}(t)\right]^{2}\right)^{3}}+\frac{1}{4} \frac{1}{\left(1+\left[u^{\prime}(-L)\right]^{2}\right)^{3}}<0,
$$

therefore, $\forall t \in[-L, L],\left|u^{\prime}(t)\right|<\left|u^{\prime}(-L)\right|$. Now we prove that Problem $I$ has got only one solution $\left({ }^{\mathrm{e}}\right)$. Let us suppose, by contradiction, that Problem $I$ admits two different solutions $u_{1}, u_{2} \in P_{1}$. From the equation

\footnotetext{
${ }^{e}$ for each $H$ (that is for each material constituting the membrane) for which the existence of the solution is verified.
} 


\section{P. DI BARBA, L. FATTORUSSO, M. VERSACI}

associated to the Problem $I$, by integration from $-L$ to $t$, we obtain, $\forall t \in$ $[-L, L]$

$$
\begin{aligned}
& u_{1}^{\prime}(t) \leq H-\frac{1}{\beta_{1} \overline{\lambda^{2}}} \int_{-L}^{t}\left[1+\left(u_{1}^{\prime}(x)\right)^{2}\right]^{3}\left[1-d^{*}-u_{1}(x)\right]^{2}, d x, \\
& u_{2}^{\prime}(t) \leq H-\frac{1}{\beta_{1} \overline{\lambda^{2}}} \int_{-L}^{t}\left[1+\left(u_{2}^{\prime}(x)\right)^{2}\right]^{3}\left[1-d^{*}-u_{2}(x)\right]^{2} d x,
\end{aligned}
$$

and, subtracting on both members, $\forall t \in[-L, L]$

$$
\begin{array}{r}
u_{1}^{\prime}(t)-u_{2}^{\prime}(t)=\frac{1}{\beta_{1} \overline{\lambda^{2}}} \int_{-L}^{t}\left\{\left[1+\left(u_{2}^{\prime}(x)\right)^{2}\right]^{3}\left[1-d^{*}-u_{2}(x)\right]^{2}-\right. \\
\left.\left[1+\left(u_{1}^{\prime}(x)\right)^{2}\right]^{3}\left[1-d^{*}-u_{1}(x)\right]^{2}\right\} d x .
\end{array}
$$

In order to evaluate the term inside the integral, let us consider the following functions:

$$
\begin{array}{r}
F(w, v)=\left[1+w^{2}\right]^{3}\left(1-d^{*}-v\right)^{2}, \\
g(t)=F\left(t w_{1}+(1-t) w_{2}, t v_{1}+(1-t) v_{2}\right)=F\left(w_{t}, v_{t}\right)
\end{array}
$$

we observe that

$$
\begin{array}{r}
g^{\prime}(t)=\frac{\partial F\left(w_{t}, v_{t}\right)}{\partial w}\left(w_{1}-w_{2}\right)+\frac{\partial F\left(w_{t}, v_{t}\right)}{\partial v}\left(v_{1}-v_{2}\right) ; \\
g(1)=F\left(w_{1}, v_{1}\right), g(0)=F\left(w_{2}, v_{2}\right), g(1)-g(0)=g^{\prime}(\xi), \xi \in(0,1),
\end{array}
$$

but

$$
\begin{array}{r}
\frac{\partial F\left(w_{\xi}, v_{\xi}\right)}{\partial w}=6\left[1+w_{\xi}^{2}\right]^{2} w_{\xi}\left(1-d^{*}-v_{\xi}\right)^{2}= \\
=6\left\{1+\left[\xi w_{1}+(1-\xi) w_{2}\right]^{2}\right\}^{2}\left[\xi w_{1}+(1-\xi) w_{2}\right]\left(1-d^{*}-v_{\xi}\right)^{2} \leq \\
\leq 6\left\{\xi\left[1+w_{1}^{2}\right]^{2}+(1-\xi)\left[1+w_{2}^{2}\right]^{2}\right\}\left[\xi w_{1}+(1-\xi) w_{2}\right]\left(1-d^{*}-v_{\xi}\right)^{2} .
\end{array}
$$

Because $w_{1} \leq H, w_{2} \leq H, v_{\xi} \leq 1$, it results that:

$$
\left|\frac{\partial F\left(w_{\xi}, v_{\xi}\right)}{\partial w}\right| \leq 24\left(1+H^{2}\right)^{2} H
$$

and

$$
\begin{aligned}
& \left|\frac{\partial F\left(w_{\xi}, v_{\xi}\right)}{\partial v}\right|=\left|-2\left[1+\left(w_{\xi}\right)^{2}\right]^{3}\left(1-d^{*}-v_{\xi}\right)\right| \leq \\
\leq & 2\left|\xi\left(1+w_{1}^{2}\right)^{3}+(1-\xi)\left(1+w_{2}^{2}\right)^{3}\right| \leq 4\left(1+H^{2}\right)^{3} .
\end{aligned}
$$




\section{Electrostatic field in terms of geometric curvature in membrane MEMS devices}

Taking into account the last inequality, by (55) and exploiting the Poincaré's inequality, $\forall t \in[-L, L]$

$$
\begin{aligned}
\left|u_{1}^{\prime}(t)-u_{2}^{\prime}(t)\right| \leq & \frac{24}{\beta_{1} \overline{\lambda^{2}}}\left(1+H^{2}\right)^{2} H \int_{-L}^{t}\left|u_{1}^{\prime}(x)-u_{2}^{\prime}(x)\right| d x+ \\
& +\frac{4}{\beta_{1} \overline{\lambda^{2}}}\left(1+H^{2}\right)^{3} \int_{-L}^{t}\left|u_{1}(x)-u_{2}(x)\right| d x \leq \\
\leq & \frac{24}{\beta_{1} \overline{\lambda^{2}}}\left(1+H^{2}\right)^{2} H \int_{-L}^{t}\left|u_{1}^{\prime}(x)-u_{2}^{\prime}(x)\right| d x+ \\
& +\frac{8 L}{\beta_{1} \overline{\lambda^{2}}}\left(1+H^{2}\right)^{3} \int_{-L}^{t}\left|u_{1}^{\prime}(x)-u_{2}^{\prime}(x)\right| d x \leq \\
& \leq c\left(H, L, \bar{\lambda}, \beta_{1}\right) \int_{-L}^{t}\left|u_{1}^{\prime}(x)-u_{2}^{\prime}(x)\right| d x .
\end{aligned}
$$

Then $\left|u_{1}^{\prime}(t)-u_{2}^{\prime}(t)\right| \leq c\left(H, L, \bar{\lambda}, \beta_{1}\right) \int_{-L}^{t}\left|u_{1}^{\prime}(x)-u_{2}^{\prime}(x)\right| d x$ from which, by Gronwall's lemma, we have, $\forall t \in[-L, L],\left|u_{1}^{\prime}(t)-u_{2}^{\prime}(t)\right| \leq 0$. Then, $\forall t \in$ $[-L, L]: u_{1}^{\prime}(t)-u_{2}^{\prime}(t)=0$, that is $u_{1}-u_{2}=$ constant, and taking into account that $u_{1}(-L)=u_{2}(-L)=u_{1}(L)=u_{2}(L)=0$, we have $u_{1}=u_{2}$.

Now we prove 2).

Let us consider $u$ as a solution of the Problem I. Setting $v(t)=u(-t)$, $\forall t \in[-L, L]$, we have that also $v$ is a solution of the this problem ${ }^{\mathrm{f}}$ and then , considering the uniqueness above proved, $v(t)=u(t), \forall t \in[-L, L]$, so that $u(t)=u(-t)$ over $[-L, L]$.

Now we prove 3 ).

Taking into account that $u$ belongs to the class $C^{2}$,the second member of the equation belongs to the class $C^{1}$ and than $u \in C^{3}([-L, L])$. By induction, we obtain that $u \in C^{\infty}([-L, L])$.

In addition, it is easy to prove that $u$ is an analytical function.

\section{Some Numerical Tests}

In this paragraph,we numerically verify that system (45) admits a solution compatible with the analytical results. The reduction of system (45) to the inequality (47) is confirmed by both simple remarks on the orders of amplitude of the involved quantities and numerical tests. In fact, owing $L_{1}=1 / 2$ and setting $\bar{\lambda}=1$, from (44) $H$ is lower than a quantity whose

\footnotetext{
$f$ In fact: $v^{\prime}(t)=-u^{\prime}(-t), v^{\prime \prime}(t)=u "(-t)$, substituting in the equation $u^{\prime \prime}(-t)=$ $-\frac{\left[1+\left(u^{\prime}(-t)\right)^{2}\right]^{3}}{\beta_{1} \overline{\lambda^{2}}}\left(1-d^{*}-u(-t)\right.$. and, in addition, as above mentioned, $v^{\prime}(-L)=-u^{\prime}(L)=$ $u^{\prime}(-L) \leq H$
} 


\section{P. DI BARBA, L. FATTORUSSO, M. VERSACI}

order of amplitude is at least $10^{2}$, then:

$$
\left\{\begin{array}{l}
1+H^{6}<\frac{H \beta_{1} \overline{\lambda^{2}}}{4 \alpha L_{1}}<\frac{10^{2} 10^{12} \overline{\lambda^{2}}}{2 \alpha} \\
1+H^{6}<\frac{\beta_{1} \bar{\lambda}^{2}}{4 L_{1}^{2}}<10^{12} \frac{\lambda^{2}}{}
\end{array}\right.
$$

and, taking into accont that $\alpha<1, \frac{\beta_{1} \overline{\lambda^{2}}}{4 L_{1}^{2}}<\frac{H \beta_{1} \overline{\lambda^{2}}}{4 \alpha L_{1}}$ so that (45) is equivalent to (47). In addition, we rewrite (61) as:

$$
\left\{\begin{array}{l}
\frac{H 10^{12} \overline{\lambda^{2}}}{2 \alpha}-H^{6}-1>0 \\
10^{12 \overline{\lambda^{2}}}\left(1-d^{*}\right)-H^{6}-1>0
\end{array}\right.
$$

and set $f_{1}(H)=\frac{H 10^{12} \overline{\lambda^{2}}}{2 \alpha}-H^{6}-1$ (continuous function). If, for example, $\overline{\lambda^{2}}=1$ and because $f_{1}(200)>0$ and $f_{1}(230)<0$ (Fig. 2), in [200,230], the existence of zeros theorem is applicable. This zero represents the sup of the set of the value of $H$ that verifies the first inequality of (62) (that is the first inequality of (45)). Therefore, applying the Newton-Raphson procedure $^{\mathrm{g}}$ (tolerance equal to 0.0001 ) we carry out $f_{1}(H)=0$ on $H=223$. Analogously, for the other inequality of (62) (Fig. 2), referred to interval $[20,140]$, with the same tolerance and $d^{*}=0$, and defining $f_{2}(H)=10^{2} \overline{\lambda^{2}}-$ $H^{6}-1$ we obtain $f_{2}(H)=0$ when $H=99$. So, to guarantee the existence of the solution of the problem, it is necessary that $\sup |H|=99$ corresponding to $88.92^{\circ}$ in dimensionless conditions. This result is comparable with the condition carried out analytically by (44). Finally, as shown in Fig. 2, when $\overline{\lambda^{2}}$ increases (towards the arrows), the bigger

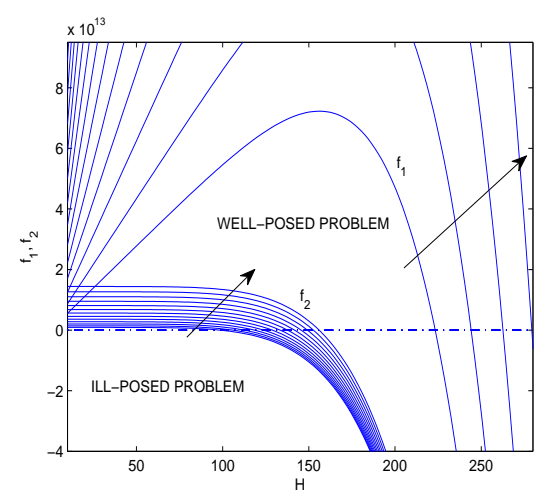
is $\sup |H|$ as higher $\overline{\lambda^{2}}$ is.

\section{Acknowledgements}

The authors wish to thank Prof. Daniele Cassani with the Department of Science and High Technology, University of Insubria (Italy) and Prof. Antonio Tarsia with the Department of Mathematics, University of Pisa (Italy) for the meaningful comments.

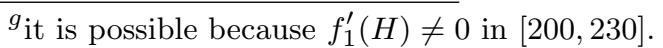


Electrostatic field in terms of geometric curvature in membrane MEMS devices

\section{REFERENCES}

1. H.C. Nathanson and W.E. Newell and R.A. Wickstrom and J.R. Lewis, The Resonant Gate Transistor, IEEE Transaction on Electron Devices, vol. 14, pp. 117-133, 1964.

2. M. Huja and M. Husak, Thermal Microactuators for Optical Purpose, Coding and Computing, pp. 137-142, 2001.

3. X. Yang and Y.C. Tai and C.M. Ho, Micro Bellow Actuators, Transducers'97, pp. 45-48, 1997.

4. S. Bhansali and A.L. Zhang and R.B. Zmood and P. Jones and D.K. Sood, Prototype Feedback Controlled Bi-Directional Actuation System for MEMS Application, Journal Microeletromechanic Systems, vol. 9, pp. 245-251, 2000.

5. A. Ludwig and E. Quandt, Giant Magnetostrictive Thin Films for Application in Microelectromechanical Systems, Journal of Applied Physics, vol. 87, pp. 4691-4695, 2000.

6. M. Elwenspoek and R. Wiegerink, Micromechanical Sensors. 2001.

7. S.D. Senturia, Microsystem Design. Kluwer Academic Publisher, 2001.

8. E. Schaffer and T Thurn-Albrecht and T.P. Russell and U. Steiner, Electrically Induced Structure Formation and Pattern Transfer, Nature, vol. 403, pp. 518-524, 2001.

9. D. Bernstein and P. Guidotti and J. Pelesko, Analytical and Numerical Analysis of Electrostatically Actuated MEMS Devices, in Proceedings of MSM 2000, pp. 489-492, 2000.

10. J.A. Pelesko and X.Y. Chen, Electrostatic Deflections of Circular Elastic Membranes, Journal of Electrostatics, vol. 57, no. 1, pp. 1-12, 2003.

11. M.O. Hasse and M.A. Hawwa and H.M.Alqahtani, Modeling the Electrostatic Deflection of a MEMS Multilayers Based Actuator, Mathematical Problems in Engineering, vol. 2013, 2013.

12. AA.VV., MEMS for Biomedical Application. Elsevier, 2012.

13. A. Folch, Introduction to Bio-MEMS. Boca Raton, 2013.

14. P. Das Payel and M. Kanoria, Magneto-Thermo-Elastic Waves in an Infinite Perfectly Conducting Elastic Solid with Energy Dissipation, $A p$ plied Mathematics and Mechanics, vol. 30, no. 2, pp. 221-228, 2009.

15. P. Di Barba and A. Lorenzi, A Magneto-Thermo-Elastic Identification Problem with a Moving Boundary in a Micro-Device, Milan Journal of Mathematics, vol. 81, no. 2, pp. 347-383, 2013.

16. R. Selvamani and P. Pommusamy, Wave Propagation in a Transversely Isotropic Magneto-Electro-Elastic Solid Bar Immersed in an Inviscid Fluid, Journal of the Egyptian Mathematical Society, vol. 24, pp. 9299, 2016. 


\section{P. DI BARBA, L. FATTORUSSO, M. VERSACI}

17. P. Di Barba and S. Wiak, Evolutionary Computing and Optimal Design of MEMS, IEEE Trans Mechatronics, vol. 20, no. 4, pp. 1660-1667, 2015.

18. P. Di Barba and M.E. Mognaschi and P. Venini and S. Wiak, Biogeography-Inspired Multiobjective Optimisation for Helping MEMS Synthesis, Archives of Electrical Engineering, in press.

19. D. Cassani and L. Fattorusso and A. Tarsia, Nonlocal Singular Problems and Application to MEMS, in Proceedings of WCE 201, 2013.

20. D. Cassani and L. Fattorusso and A. Tarsia, Nonlocal Dynamic Problems with Singular Nonlinearities and Application to MEMS, Progress in Nonlinear Differential Equations and their Applications, vol. 85, pp. 185-206, 2014.

21. D. Cassani and A. Tarsia, Periodic Solutions to Nonlocal MEMS Equations, Discrete and Continuousd Dynamical Systems - Serie S, vol. 9, no. 3, pp. 631-642, 2016.

22. S.E. Lyshevski, MEMS and NEMS: Systems, Devices and Structures. Boca Raton, 2002.

23. D. Cassani and M. d'O and N. Ghoussoub, On a Fourth Order Elliptic Problem with a Singular Nonlinearity, Nonlinear Studies, vol. 9, pp. 189-209, 2009.

24. D. Cassani and B. Kaltenbacher and A. Lorenzi, Direct and Inverse Problem Related to MEMS, Inverse Problems, vol. 25, 2009.

25. C. Cowan and P. Esposito and N. Ghoussoub and Mordifam, The Critical Dimension for a Fourth Order Elliptic Problem with Singular Nonlinearity, Arch. Ration. Mech. Anal., vol. 198, pp. 763-787, 2010.

26. C. Cowan and P. Esposito and N. Ghoussoub, Regularity of Extremal Solutions in Fourth Order Nonlinear Eigenvalue Problems on General Domains, Discrete and Continuous Dynamical Systems, vol. 28, no. 2, pp. 1-19, 2010.

27. P. Laurencot and C. Walker, A Stationary Free Boundary Problem Modeling Electrostatic MEMS, Arch. Ration. Mech. Anal., vol. 207, no. 1, pp. 139-158, 2013.

28. J.A. Pelesko and D.H. Bernstein, Modeling MEMS and NEMS. Chapman \& Hall, 2003.

29. J.D. Jackson, Classical Electrodynamics. John Wiley, 1975.

30. G. Barozzi and F. Gasparini, Fondamenti di Elettrotecnica: Elettromagnetismo. UTET, 1989.

31. P.B. Bayley and L.F. Shampine and P.E. Waltmman, Nonlinear Two Point Boundary Value Problems. Academic Press, 1968. 